\title{
基于环 $\mathbb{Z}_{n}$ 上圆雉曲线的 $\mathrm{QV}$ 签名方案
}

\author{
王标 (1) * 方颖珏 (2), 林宏刚 (3), 李轶 (1)
}

\author{
(1) 国际关系学院信息科技系, 北京 100091 \\ (2) 深圳大学数学与计算科学学院, 深圳 518060 \\ (3) 成都信息工程学院网络工程系, 成都 610225 \\ *E-mail: wangbiao@uir.cn
}

收稿日期: 2007-08-26; 接受日期: 2008-10-22

国家自然科学基金 (批准号: 10128103) 和国家保密通信重点实验室基金 (批准号: 51436010505sc0101) 资助项目

\begin{abstract}
摘要 经典 $\mathrm{RSA}$ 算法易受小指数攻击, 并具有同态性. 环 $\mathbb{Z}_{n}$ 上椭圆曲线 $E_{n}(a, b)$ 上的 $\mathrm{KMOV}$ 签名方案克服了小指数攻击, 但是仍有同态性. $E_{n}(a, b)$ 上的 $\mathrm{QV}$ 签名方 案克服小指数攻击及同态性, 但要求 $E_{n}(a, b)$ 上存在阶为 $M_{n}=\left\{\sharp E_{p}(a, b), \sharp E_{q}(a, b)\right\}$ 的点, 而这一条件不是所有 $E_{n}(a, b)$ 都能满足, 且 $E_{n}(a, b)$ 上的计算较为复杂. 文中进 一步研究环 $\mathbb{Z}_{n}$ 上圆锥曲线 $C_{n}(a, b)$ 及其性质, 得到用以构建数字签名方案的几个关键 定理和推论, 指出 $C_{n}(a, b)$ 上总是存在阶为 $M_{n}=\left\{\sharp E_{p}(a, b), \sharp E_{q}(a, b)\right\}$ 的点, 在此基础 上, 提出一个基于 $C_{n}(a, b)$ 上的 $\mathrm{QV}$ 签名方案, 新方案保留原方案在 $E_{n}(a, b)$ 上不具同 态性的优点, 在同等安全条件下, 其明文嵌入、阶的计算、逆元的计算、点的运算都比 $E_{n}(a, b)$ 上容易, 特别是, 新的 $\mathrm{QV}$ 签名方案对于一般环上圆锥曲线均可行, 这对 $\mathrm{QV}$ 方案的应用有积极意义.
\end{abstract}

\section{关键词}

数字签名

环 $\mathbb{Z}_{n}$ 上圆雉曲线

$\mathrm{RSA}$

小指数攻击

KMOV

QV

\section{1 引言}

由于建立在经典 RSA 算法上的签名方案容易 受到小加密指数和小解密指数攻击等威胁, 1992 年 Koyama, Maurer, Okamoto, Vanstone 在文献 [1] 中 提出了基于环 $\mathbb{Z}_{n}$ 上椭圆曲线上的数字签名方案, 简称 $\mathrm{KMOV}$ 方案. 它的优点是能够抵抗小指数攻 击, 缺点是该方案具有同态性 ${ }^{[2]} .2000$ 年, $\mathrm{Qu}$ 和 Vanstone $^{[2]}$ 提出一个没有同态性的环上椭圆曲线的 数字签名方案, 简称QV方案. 2003 年, 朱文余和孙 琦在文献 $[3,4]$ 中, 对环 $\mathbb{Z}_{n}$ 上椭圆曲线 $E_{n}(a, b)$ 的基 本性质进行了深入的研究, 并进一步研究了 $\mathrm{KMOV}$ 和 $\mathrm{QV}$ 签名方案, 指出如果 $E_{n}(a, b)$ 不存在阶为 $M_{n}=\left\{\sharp E_{p}(a, b), \sharp E_{q}(1, b)\right.$ 的点, 则 $E_{n}(a, b)$ 上的 $\mathrm{QV}$ 方案就不能使用, 文献 [3] 中给出了一个不存在阶为 $M_{n}$ 的点的例子, 说明 $\mathrm{QV}$ 签名方案对一般 $\mathbb{Z}_{n}$ 上的 椭圆曲线不一定可行, 这使得该签名方案的应用有较
大限制.

1998 年, 曹珍富 ${ }^{[5]}$ 提出了基于有限域上圆雉曲 线的公钥密码体制, 随后, 他又提出了 RSA 的圆锥 曲线模拟 ${ }^{[6]} ; 2005$ 年, 孙琦等在文献 [7] 中定义并 系统研究了环 $\mathbb{Z}_{n}$ 上的圆雉曲线 $C_{n}(a, b)$, 提出了基 于 $C_{n}(a, b)$ 的公钥密码体制; 王标等在文献 [8] 中 进一步研究了环上圆雉曲线密码体制的性质, 并指 出 $C_{n}(a, b)$ 上 RSA 型的公钥密码体制, 不仅保留了 $E_{n}(a, b)$ 上的优点, 而且计算更简单, 具有应用前景.

本文首先进一步研究环上圆雉曲线 $C_{n}(a, b)$ 的性 质, 得到 $C_{n}(a, b)$ 上总是存在阶为 $M_{n}$ 的基点 $G$ (定 理 2 及其推论), 提出了 $C_{n}(a, b)$ 上的 $\mathrm{QV}$ 签名方 案, 该签名方案与 $E_{n}(a, b)$ 上的方案相比, 不仅保留 了抗小指数 $e$ 攻击和不具同态性的优点, 还证明在 Wiener 定理条件下, 方案是安全的; 同时, 与 $E_{n}(a, b)$ 上的方案相比, 安全强度不变的情况下, 计算更加简 
单; 特别地, 由于 $C_{n}(a, b)$ 上的阶为 $M_{n}$ 的基点 $G$ 一 定存在, 新方案在一般的 $C_{n}(a, b)$ 上均可行, 这对 $\mathrm{QV}$ 方案的实现有积极意义.

\section{$2 \mathrm{KMOV}$ 和 $\mathrm{QV}$ 签名方案}

设 $\mathbb{Z}_{n}$ 是一个模 $n$ 的剩余类环, 考虑 $\mathbb{Z}_{n}$ 上的三 次曲线 $E_{n}(a, b)$ :

$$
y^{2} \equiv x^{3}+a x+b(\bmod n),
$$

其中 $n=p q\left(p, q\right.$ 为奇素数). 当 $\left(4 a^{3}+27 b^{2}, n\right)=1$ 时, 射影曲线 $E_{n}(a, b)$ 定义了一条环 $\mathbb{Z}_{n}$ 上的椭圆曲线.

需要注意的是, 与有限域上椭圆曲线 $E_{p}(a, b)$ 不 同, 环上椭圆曲线存在两个问题: 一是环上逆元运算 只有当其与 $n$ 互素的时候才行, 于是对于某些点在 $E_{n}(a, b)$ 上的运算不一定有意义; 二是 $E_{n}(a, b)$ 不是 一个群. 这两个问题对 $E_{n}(a, b)$ 上的密码体制的应 用实现有一定的影响.

\section{$2.1 \mathrm{KMOV}$ 签名方案及安全性分析}

发送方 Alice 首先选择一条椭圆曲线 $E_{n}(a, b)$ 满 足 $\left(4 a^{3}+27 b^{2}, n\right)=1, n=p q, p, q$ 为两个不同的大 素数. 然后计算 $\sharp E_{p}(a, b), \sharp E_{q}(a, b)$. Alice 选择加密 密钥 $e$, 其中

$$
\left(e, \sharp E_{p}(a, b)\right)=1, \quad\left(e, \sharp E_{q}(a, b)\right)=1 .
$$

计算解密密钥 $d$ 满足:

$$
e d \equiv 1\left(\bmod 1 \mathrm{~cm} \quad\left(\sharp E_{p}(a, b), \sharp E_{q}(a, b)\right) .\right.
$$

于是 Alice 的公开密钥为 $n, a, b, e$; 私钥为 $d, p, q$, $\sharp E_{p}(a, b), \sharp E_{q}(a, b)$.

Alice 签名消息 $m(0 \leqslant m<n)$ 如下:

首先将明文 $m$ 嵌入到椭圆曲线上一点 $P(m)=$ $(x, y)$, 则 $x$ 为大于等于 $m$ 的最小正整数使得 $x^{3}+$ $a x+b$ 模 $n$ 的二次剩余, $y$ 定义为 $x^{3}+a x+b$ 模 $n$ 的一个平方根.

计算 $Q=(s, t)=d P(m), m$ 的签名信息为 $(s, t)$.

签名验证: $P(m)=e Q$, 若等式成立, 则接受签 名, 否则拒绝.

在文献 $[2,3]$ 的基础上, 对 KMOV 方案的安全性 分析如下:

1) 算法的安全性是建立在大数分解的困难性的 基础上 ${ }^{[2]}$, 即如果我们不知道 $n$ 的完全分解, 很难从 $n, a, b, e$ 得到 $d$.
2) KMOV 方案在抵抗低加密指数攻击和抵抗低 解密指数攻击等方面比经典 RSA 算法安全 ${ }^{[3]}$.

3) KMOV 方案具有同态性 ${ }^{[2]}$, 原因是针对不同 消息 $m_{1}$ 和 $m_{2}$, 对应椭圆曲线上点为 $P_{1}$ 和 $P_{2}$, 对应 的签名分别为 $Q_{1}$ 和 $Q_{2}$, 则有 $Q_{1}=d P_{1}$ 和 $Q_{2}=d P_{2}$, 因此有 $Q_{1}+Q_{2}=d P_{1}+d P_{2}=d\left(P_{1}+P_{2}\right)$.

\section{$2.2 E_{n}(a, b)$ 上的 $\mathrm{QV}$ 签名方案}

针对 $\mathrm{KMOV}$ 方案的同态性, 2000 年, $\mathrm{Qu}$ 和 Vanstone $^{[2]}$ 提出了一个没有同态性的环上椭圆曲线 的数字签名方案, 我们称为 $\mathrm{QV}$ 签名方案. 方案具体 内容见文献 [2].

需要注意的是: 如果 $E_{n}(a, b)$ 不存在阶为 $M_{n}$ 的 点, 则 $E_{n}(a, b)$ 上的 $\mathrm{QV}$ 方案就不能实现, 文献 [3] 给 出了一个例子, $E_{n}(-1,0)(n=p q, p=211, q=163)$, 不存在阶为 $M_{n}$ 的点. 因此 $\mathrm{QV}$ 方案只能在部分椭 圆曲线上实现

这对环上椭圆曲线密码的应用产生较大限制.

\section{3 环 $\mathbb{Z}_{n}$ 上圆雉曲线 $C_{n}(a, b)$ 及其 $\mathrm{QV}$ 签 名方案}

\section{$3.1 C_{n}(a, b)$ 及其性质}

设 $\mathbb{Z}_{n}$ 是一个模 $n$ 的剩余类环, 我们定义环 $\mathbb{Z}_{n}$ 上的圆雉曲线是同余方程

$$
y^{2} \equiv a x^{2}-b x(\bmod n)
$$

在 $\mathbb{Z}_{n}$ 上的解 $(x, y)$ 的集, 这里 $n=p q, p, q$ 为两个不 同的奇素数, $(a, n)=(b, n)=1$. 详细内容参见文献 $[7,8]$.

显然 $O=(0,0) \in C_{n}(a, b)$. 记 $C_{n}(a, b)$ (即同余 方程 (1) 的解集) 为:

$$
\begin{aligned}
C_{n}(a, b)=\left\{(x, y) \in \mathbb{Z}_{n} \times \mathbb{Z}_{n}\right. \\
\left.\mid y^{2}=a x^{2}-b x \equiv 0(\bmod n)\right\},
\end{aligned}
$$

文献 [7] 以坐标的方式给出 $C_{n}(a, b)$ 中全部有理点的 表示

$$
C_{n}(a, b)=C_{1} \cup C_{2} \cup C_{3} \cup O,
$$

其中

$$
\begin{aligned}
& C_{1}=\left\{P_{1}(t)=\left(b\left(a-t^{2}\right)^{-1}, t b\left(a-t^{2}\right)^{-1}\right),\right. \\
&\left.\left(a-t^{2}, n\right)=1, \forall t \in \mathbb{Z}_{n}\right\} ; \\
& C_{2}=\left\{P_{2}(t)=\left(p p^{-1} b\left(a-t^{2}\right)^{-1}, p p^{-1} b t\left(a-t^{2}\right)^{-1}\right),\right.
\end{aligned}
$$




$$
\begin{aligned}
&\left(a-t^{2}, q\right)=1, \forall t \in \mathbb{Z}_{q}, p p^{-1} \equiv 1(\bmod q), \\
&\left.\left(a-t^{2}\right)\left(a-t^{2}\right)^{-1} \equiv 1(\bmod q)\right\} ; \\
& C_{3}=\left\{P_{3}(t)=\left(q q^{-1} b\left(a-t^{2}\right)^{-1}, q q^{-1} b t\left(a-t^{2}\right)^{-1}\right),\right. \\
&\left(a-t^{2}, p\right)=1, \forall t \in \mathbb{Z}_{p}, q q^{-1} \equiv 1(\bmod p), \\
&\left.\left(a-t^{2}\right)\left(a-t^{2}\right)^{-1} \equiv 1(\bmod p)\right\} .
\end{aligned}
$$

文献 [7] 定义环 $\mathbb{Z}_{n}$ 上的圆雉曲线 $C_{n}(a, b)$ 中 的加法运算 $\oplus$ : 对任意 $P=\left(x_{1}, y_{1}\right) \in C_{n}(a, b)$ 和 $Q=\left(x_{2}, y_{2}\right) \in C_{n}(a, b)$, 定义 $P \oplus Q$ 如下:

1) 当 $P \neq Q$ 时, $P \oplus Q$ 的定义为:

(1) 若 $\left(x_{2}-x_{1}, n\right)=1$, 则 $P \oplus Q=P_{1}(t) \in C_{1}$, 其中 $t=\frac{y_{2}-y_{1}}{x_{2}-x_{1}} \bmod n$.

(2) 若 $\left(x_{2}-x_{1}, n\right)=p$, 则 $P \oplus Q=P_{2}(t) \in C_{2}$, 其中 $t=\frac{y_{2}-y_{1}}{x_{2}-x_{1}} \bmod q$.

(3) 若 $\left(x_{2}-x_{1}, n\right)=q$, 则 $P \oplus Q=P_{3}(t) \in C_{3}$, 其中 $t=\frac{y_{2}-y_{1}}{x_{2}-x_{1}} \bmod p$.

(4) 若 $\left(x_{2}-x_{1}, n\right)=n$, 则 $P \oplus Q=O$.

2) 当 $P=Q$ 时, $P \oplus Q=2 P=2\left(x_{1}, y_{1}\right)$ 的定义 为:

(5) 若 $\left(y_{1}, n\right)=1$, 则 $2 P=P_{1}(t) \in C_{1}$, 其中 $t=\frac{2 a x_{1}-b}{2 y_{1}} \bmod n$.

(6) 若 $\left(y_{1}, n\right)=p$, 则 $2 P=P_{2}(t) \in C_{2}$, 其中 $t=\frac{2 a x_{1}-b}{2 y_{1}} \bmod q$.

(7) 若 $\left(y_{1}, n\right)=q$, 则 $2 P=P_{3}(t) \in C_{3}$, 其中 $t=\frac{2 a x_{1}-b}{2 y_{1}} \bmod p$.

(8) 若 $\left(y_{1}, n\right)=n$, 则 $2 P=O$.

定理 $\mathbf{1}^{[7]}$ 环 $\mathbb{Z}_{n}$ 上的圆雉曲线 $C_{n}(a, b)$, 对于定 义的加法 $\oplus$ 构成一个有限交换群, 记为 $\left(C_{n}(a, b), \oplus\right)$.

$C_{n}(a, b)$ 在定义的加法 $\oplus$ 下构成一个有限交换 群, 这使得构建环上圆锥曲线公钥密码体制成为可 能.

设 $A \in C_{n}(a, b)$, 称使 $k A=O$ 成立的最小正整 数 $k$ 为 $A$ 的阶, 记为 $o(A)$. 我们得到以下定理及其 推论.

定理 2 对 $\forall A=(x, y) \in C_{n}(a, b), A$ 在 $C_{p}(a, b) \times C_{q}(a, b)$ 中有唯一的点 $\left(A_{p}, A_{q}\right)$ 与之对应, 且点 $A$ 的阶 $o(A)=\left\{o\left(A_{p}\right), o\left(A_{q}\right)\right\}$, 符号 $\{$,$\} 表示最$ 小公倍数.

证明 设 $o(A)=k$, 满足 $k A=O$ 的最小正整 数 $k$, 即 $k A_{p}=O_{p}, k A_{q}=O_{q}$ 同时成立的最小正整
数 $k$.

由于 $o\left(A_{p}\right)\left|k, o\left(A_{q}\right)\right| k$, 所以 $k=\left\{o\left(A_{p}\right), o\left(A_{q}\right)\right\}$.

设 $p, q$ 为两个不同的大素数, $n=p q$, 当 $\left(\frac{a}{p}\right)=$ $\left(\frac{a}{q}\right)=-1$ 时, 则有 $\sharp C_{n}(a, b)=(p+1)(q+1)$, 且设 $p+1=2 r, q+1=2 s$, 其中 $r, s$ 也为素数. 于是 在 $C_{p}(a, b)$ 中有阶为 2 的点, 也有阶为 $r$ 的点, 故有 阶为 $2 r$ 的点, 即此时 $C_{p}(a, b)$ 为 $2 r$ 阶循环群; 同理 $C_{q}(a, b)$ 为 $2 s$ 阶循环群. 设 $G_{p}$ 和 $G_{q}$ 分别为 $C_{p}(a, b)$ 和 $C_{q}(a, b)$ 的生成元, 则 $\left[G_{p}, G_{q}\right] \stackrel{\phi}{\longrightarrow} G, G \in C_{n}(a, b)$. 由定理 2 知 $G$ 的阶为 $M_{n}=2 r s$. 于是我们有以下推 论.

推论 1 设 $n=p q, p, q$ 是两个不同的大素数, 满足 $\left(\frac{a}{p}\right)=\left(\frac{a}{q}\right)=-1$ 时, 且 $p+1=2 r, q+1=2 s$, 其 中 $r, s$ 也是素数, 则曲线 $C_{n}(a, b)$ 中存在一个点 $G$, 其阶 $M_{n}=2 r s$, 且 $\left(C_{n}(a, b), \oplus\right)$ 中任意一元的阶整 除 $M_{n}$.

推论 $\mathbf{2}$ 设 $n=p q, p, q$ 是两个不同的大素数, $\left(\frac{a}{p}\right)=\left(\frac{a}{q}\right)=-1, p+1=2 r, q+1=2 s$, 其中 $r, s$ 是 素数, 则有

1) 当 $a-b \equiv 1(\bmod n)$ 时, 则

$$
G= \begin{cases}P_{1}(1), & \text { 如果 }(1,1) \text { 是 } C_{p}(a, b) \text { 的生成元 } \\ & \text { 或 }(1,1) \text { 是 } C_{q}(a, b) \text { 的生成元; } \\ P_{1}(a), & \text { 如果 }(1,1) \text { 是 } C_{p}(a, b) \text { 的 } r \text { 阶元 } \\ & \text { 或 } \left.(1,1) \text { 是 } C_{q}(a, b)\right) \text { 的 } s \text { 阶元. }\end{cases}
$$

2) 当 $a-b \equiv 4(\bmod n)$ 时, 则

$$
G= \begin{cases}P_{1}(2), & \text { 如果 }(1,2) \text { 是 } C_{p}(a, b) \text { 的生成元 } \\ & \text { 或 }(1,2) \text { 是 } C_{q}(a, b) \text { 的生成元; } \\ P_{1}(a / 2), & \text { 如果 }(1,2) \text { 是 } C_{p}(a, b) \text { 的 } r \text { 阶元 } \\ & \text { 或 } \left.(1,2) \text { 是 } C_{q}(a, b)\right) \text { 的 } s \text { 阶元. }\end{cases}
$$

证明 本文只证明 1). 对于 2), 类似可证.

由于 $a-b \equiv 1(\bmod n)$, 显然 $x=y=1$ 是 1$)$ 的 解, 即 $(1,1) \in C_{n}(a, b)$; 同理 $(1,1) \in C_{p}(a, b),(1,1) \in$ $C_{q}(a, b)$.

由于 $C_{p}(a, b)$ 和 $C_{q}(a, b)$ 均为循环群, 有

$$
\begin{aligned}
& \sharp C_{p}(a, b)=p+1=2 r, \\
& \sharp C_{q}(a, b)=q+1=2 s
\end{aligned}
$$

若 $(1,1)$ 是 $C_{p}(a, b)$ 的生成元或 $(1,1)$ 是 $C_{q}(a, b)$ 的 生成元, 则 $(1,1)$ 在 $C_{p}(a, b)$ 中的阶为 $2 r$ 或 $(1,1)$ 在 
$C_{q}(a, b)$ 的阶为 $2 s$, 由定理 2 知, $(1,1)$ 在 $C_{n}(a, b)$ 中 的阶为 $2 r s$, 即 $G=P_{1}(1)$.

由于 $\left(b a^{-1}, 0\right) \in C_{n}(a, b)$, 易知 $\left(b a^{-1}, 0\right)$ 是 $C_{p}(a, b)$ 的 2 阶点, 也是 $C_{q}(a, b)$ 的 2 阶点.

如果 $(1,1)$ 是 $C_{p}(a, b)$ 的 $r$ 阶元或 $(1,1)$ 是 $C_{q}(a, b)$ 的 $s$ 阶元, 那么在 $C_{p}(a, b)$ 中, $(1,1) \oplus$ $\left(b a^{-1}, 0\right)=P(1) \oplus P(0)=P(a)=A_{p}$ 的阶为 $2 r$ 或在 $C_{q}(a, b)$ 中, $(1,1) \oplus\left(b a^{-1}, 0\right)=P(1) \oplus P(0)=P(a)=$ $A_{q}$ 的阶为 $2 s$. 设 $\left[A_{p}, A_{q}\right] \stackrel{\phi^{-1}}{\longrightarrow} A$, 故 $A=P_{1}(a)$. 于 是由定理 2 可知 $P_{1}(a)$ 的阶为 $2 r s$, 即 $P_{1}(a)=G$. 这 就证明了 1).

\section{$3.2 C_{n}(a, b)$ 上的 $\mathrm{QV}$ 签名方案}

由定理 2 及其推论可知, $\mathrm{QV}$ 方案的实现条件在 $C_{n}(a, b)$ 上是容易满足的, 这为 $\mathrm{QV}$ 方案在圆雉曲线 上的实现奠定了理论基础.

$C_{n}(a, b)$ 上的 $\mathrm{QV}$ 签名方案设计如下:

1) 密钥管理中心建立如下:

选择一条圆雉曲线 $y^{2} \equiv a x^{2}-b x(\bmod n)$, 其 中 $a, b \in \mathbb{Z}_{n},(a, n)=(b, n)=1 . n=p q, p$ 和 $q$ 为 两个大素数, 满足 $\left(\frac{a}{p}\right)=\left(\frac{a}{q}\right)=-1$ 时, $\sharp C_{n}(a, b)=$ $(p+1)(q+1)$, 且 $p+1=2 r, q+1=2 s$, 其中 $r, s$ 也 是素数, 将此圆雉曲线记为 $C_{n}(a, b)$.

$G$ 为基点且由推论知一定存在, 并通过推论 2 的 方法获得, 其阶为 $M_{n}=2 r s$.

公开: $n, a, b, G$.

保密: $p, q, M_{n}$.

密钥管理中心计算如下:

$I_{A}$ 为散列用户 $A$ 的公开信息, 且 $\left(I_{A}, M_{n}\right)=1$. 中心计算 $D_{A}$ 满足 $I_{A} D_{A} \equiv 1\left(\bmod M_{n}\right), S_{A}=$ $-D_{A} G$, 所以, $I_{A} S_{A} \oplus G=O$.

将 $\left(I_{A} \| S_{A}\right)$ 传给用户 $A$. 其中 $I_{A}$ 为用户 $A$ 的 公开钥, $S_{A}$ 为用户 $A$ 的私钥.

2) 签名消息 $m$.

(1) 随机地选择 $C_{n}(a, b)$ 的一个点 $R$, 计算 $C_{1}=$ $I_{A} R$;

(2) 计算 $r=\operatorname{hash}\left(m \| C_{1}\right)$;

(3) 计算 $S=r S_{A} \oplus R$;

(4) $(m\|r\| S)$ 为 Alice 对 $m$ 的签名消息.

3) 签名验证.

(1) 计算 $V=I_{A} S \oplus r G$ 和 $\operatorname{hash}(m \| V)$;
(2) 签名有效充分必要条件是 $r=\operatorname{hash}(m \| V)$.

\section{$3.3 C_{n}(a, b)$ 上 $\mathrm{QV}$ 签名方案的数值模拟}

为方便验算, 本文采用笔算的数值实例, 同时使 用了标准二进制表示法 $(\mathrm{NAF})^{[9]}$.

1) 密钥管理中心建立如下:

选取圆雉曲线 $C_{65}: y^{2} \equiv 2 x^{2}-x(\bmod 65)$, 即 $a=2, b=1, n=65, p=5, q=13$, 满足 $\left(\frac{a}{p}\right)=\left(\frac{2}{5}\right)=-1,\left(\frac{a}{q}\right)=\left(\frac{2}{13}\right)=-1 . r=(p+1) / 2=$ $3, s=(q+1) / 2=7$, 所以 $M_{n}=2 r s=42$.

由于 $a-b=2-1 \equiv 1(\bmod 65)$, 满足推论 2 的 条件, $(1,1)$ 在 $C_{5}(2,1)$ 中的阶为 $r=3$, 于是由推论 2 , 可取 $C_{65}(2,1)$ 中的基点 $G$ 为: $P_{1}(2)=(32,64)$.

公开: $C_{65}(2,1), G=(32,64), n=65$;

保密: $M_{n}=42, p=5, q=13$.

给用户 $A$ 分发私钥:

假若散列用户 $A$ 的公开信息 $I_{A}=17$, 满足 $(17,42)=1$. 中心计算 $D_{A} \equiv I_{A}^{-1}\left(\bmod M_{n}\right)=$ $5, S_{A}=-D_{A} G=-5 P_{1}(2)=-P_{1}(3)$.

将 $\left(I_{A}, S_{A}\right)=\left(17,-P_{1}(3)\right)$ 传给用户 $A$, 其中 $I_{A}=17$ 为用户 $A$ 的公开钥, $S_{A}=-P_{1}(3)$ 为用户 $A$ 的私钥.

2) 签名消息 $m=12$ :

(1) 随机的选择 $C_{65}(2,1)$ 的一个点 $R=$

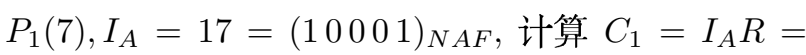
$17 P_{1}(7)=P_{1}(7) \oplus 2^{4} P_{1}(7)=P_{1}(23)$;

(2) 计算 $r=\operatorname{hash}\left(m, C_{1}\right)=\operatorname{hash}(12,23)=12$, 其中 hash 函数取选取奇数位上的数字组成其函数 值，在实际应用中，应该使用专门的 hash 函数，如 SHA 算法:

(3) 计算 $S=r S_{A} \oplus R=12 P_{1}(-3) \oplus P_{1}(7)=$ $P_{1}(50)$;

(4) $(m\|r\| S)$ 为 $A$ 对 $m=12$ 的签名消息.

3) 签名验证:

(1) 计算 $V=I_{A} S \oplus r G=17 P_{1}(50) \oplus 12 P_{1}(2)=$ $P_{1}(2), \operatorname{hash}(m, V)=\operatorname{hash}(12,2)=12$;

(2) 因此, $r=h a s h(m, V)$ 故签名是有效的.

\section{$3.4 C_{n}(a, b)$ 上 $\mathrm{QV}$ 签名方案的性能分析}

1) 新方案安全性是建立在大数分解困难性基础 上, 同时也基于圆雉曲线上离散对数困难性问题 ${ }^{[7]}$. 
2) 新方案继承了 $E_{n}(a, b)$ 上 $\mathrm{QV}$ 方案不具同 态性的优点, 并能够对抗同模攻击等威胁, 证明类似 $E_{n}(a, b)$ 上 ${ }^{[8]}$.

$3)$ 与 $E_{n}(a, b)$ 上的 $\mathrm{QV}$ 方案相比较, 新方案的 明文嵌入, 阶的计算, 逆元的计算, 点的运算都比较 容易, 也就是在同等安全条件下, 新方案实现的计算 量要少得多 ${ }^{[7,8]}$.

4) 新方案能够抵抗小指数 $I$ 攻击的 Hastad 算 法 ${ }^{[10]}$, 证明类似文献 [1] 中 $E_{n}(a, b)$ 上的. 这使得新 方案比经典 RSA 签名算法在抗小指数 $e$ 攻击方面更 安全.

5) 新方案在抗小指数 D 攻击方面: 在满足 Wiener 定理的条件下, 方案仍是安全的, 以下是相关内 容及证明:

定理 $3^{[11]}$ (Wiener) 设 $n=p q,\langle n, e\rangle$ 代表一 个 RSA 用户的公开信息, $e$ 是加密指数, $d$ 是私 钥指数. 如果素数 $p, q$ 满足 $q<p<2 q$ 以及 $1<d<(1 / 3) n^{1 / 4}$, 则通过 $\langle n, e\rangle$, 可有效的求出 $d$.

Wiener 定理的证明初等而简短, 可参见文献 $[11,12]$. 这里给出证明的要点. 由定理所设条件可 得 $p+q-1<3 \sqrt{n}$, 又设 $1<e<\varphi(n), e d=$ $k \varphi(n)+1, k \in \mathbb{Z}$, 故 $k<d$, 以及

$$
\left|\frac{e}{n}-\frac{k}{d}\right|<\frac{1}{2 d^{2}} \text {. }
$$

注意到 $(k, d)=1$, 故知 $d$ 是 $\frac{e}{n}$ 的有限简单连分数展 式中某个渐进分数的分母, 因此可有效求出 $d$.

定理 4 设 $n=p q,\langle n, I, a, b\rangle$ 代表一个 $C_{n}(a, b)$ 上的 $\mathrm{QV}$ 签名方案的公开信息, $I$ 是签名指数, $D$ 是验证指数, 如果素数 $p, q$ 满足 $q<p<2 q$ 以及 $1<D<\frac{1}{3} n^{1 / 4}$, 则方案仍是安全的.

证明 由签名算法知 $M_{n}=2 r s$, 其中 $p+1=$ $2 r, q+1=2 s, r, s$ 是两个素数, $I D \equiv 1\left(\bmod M_{n}\right)$, 可知 $I D=k M_{n}+1, k \in \mathbb{Z}, k M_{n}<I D$. 不妨设 $1<I<M_{n}$, 故知 $k<D$. 再由 $q<p<2 q$ 可得 $n=p q>q^{2}$. 于是 $q<\sqrt{n}, p<2 \sqrt{n}, p+q<3 \sqrt{n}$, 且

$$
\begin{aligned}
\left|\frac{I}{n}-\frac{k}{D}\right| & =\left|\frac{I D-n k}{n D}\right| \\
& =\left|\frac{1+\left(k M_{n}-n k\right)}{n D}\right| \\
& =\left|\frac{1+k \frac{(p+1)(q+1)}{2}-n k}{n D}\right|
\end{aligned}
$$

$$
\begin{aligned}
& =\left|\frac{2+k(p+1)(q+1)-2 n k}{2 n D}\right| \\
& =\left|\frac{2+k(p+q+1)-n k}{2 n D}\right| \\
& =\frac{k(n-(p+q+1))-2}{2 n D} .
\end{aligned}
$$

下面我们来证明, 当 $q>5$ 时, (3) 式不能成立, 即不等式

$$
\frac{k(n-(p+q+1))-2}{2 n D}<\frac{1}{2 D^{2}}
$$

不能成立. 如果 $(3 \mathrm{~b})$ 成立, 则有 $D k(n-(p+q+1))<$ $n+2 D$.

由于 $D \geqslant 2, k \geqslant 1$, 上式给出 $2(n-(p+q+1))<$ $n+2 D$, 即得 $n=p q<2(p+q+1+D)$. 注意到 $q<p$ 和 $D<\left(\frac{1}{3}\right) n^{1 / 4}$, 因此 $n=p q<2\left(p+q+1+\left(\frac{1}{3}\right) n^{1 / 4}\right)<$ $2\left(2 p+1+\left(\frac{1}{3}\right) \sqrt{p}\right)$, 即 $q<2\left(2+\frac{1}{p}+\left(\frac{1}{3 \sqrt{p}}\right)\right)$ 与 $q>5$ 矛盾.

因此(3b)不能成立. 即 $q>5$ 时, 方案是安全的.

文献 [13] 还指出 $E_{n}(a, b)$ 的 $\mathrm{QV}$ 方案能对抗 Wiener 攻击, 该性质自然继承到 $C_{n}(a, b)$ 上.

注意: 文献 [13] 和本文所提及的 Wiener 定理是 均只指 1990 年 Wiener 本人提出的攻击方法.

$6)$ 特别地, $E_{n}(a, b)$ 上 $\mathrm{QV}$ 方案, 只有当 $E_{n}(a, b)$ 中存在阶为 $M_{n}$ 的点时才能使用, 文献 [3] 给出了一 个例子: $E_{n}(-1,0)(n=p q, p=211, q=163)$, 不存在 阶为 $M_{n}$ 的点, 因此 $E_{n}(a, b)$ 上的 $\mathrm{QV}$ 方案只能在部 分椭圆曲线上实现.

而在 $C_{n}(a, b)$ 上, 由定理 2 及其推论 1 和 2 知, 在 $C_{n}(a, b)$ 上, 总是存在阶为 $M_{n}$ 的点, 推论 2 还给出了 获得阶为 $M_{n}$ 的基点 $G$ 的一类算法, 因此, $C_{n}(a, b)$ 上的 $\mathrm{QV}$ 方案的圆雉曲线选择没有类似 $E_{n}(a, b)$ 上 的限制条件.

换而言之, $C_{n}(a, b)$ 上的 $\mathrm{QV}$ 签名方案对于一般 环上圆锥曲线均可行.

7) 方案中选择 $p+1=2 r, q+1=2 s$ 是为了防止 Pohlig-Hellman 攻击, 类比证明及相关内容具体可以 参见文献 [14].

\section{4 结论}

环上圆锥曲线公钥密码体制是一类较新型的曲 线类公钥密码体制, 曲线上的 $\mathrm{QV}$ 算法 (包括 $C_{n}(a, b)$ 
和 $\left.E_{n}(a, b)\right)$, 均克服了经典 RSA 算法和 $\mathrm{KMOV}$ 方 案所具有的同态性, 并能够抵抗针对小加密指数 $e$ 和 小解密指数 $d$ 的一些攻击算法. 本文定理 4 给出了 新方案在 Wiener 定理条件下是安全的证明.

环上圆雉曲线RSA型公钥密码体制与环上椭圆 曲线RSA型密码体制相比, 其安全基础是一致的, 而 且 $C_{n}(a, b)$ 上的公钥密码算法计算更加简单, 尤其反 映在明文嵌入、阶的计算、逆元的计算、点的运算等 方面, 这些对密码算法的设计和应用有积极意义.

由于 $E_{n}(a, b)$ 上的 $\mathrm{QV}$ 签名方案要求该椭圆曲
线上存在阶为 $M_{n}=\left\{\sharp E_{p}(a, b), \sharp E_{q}(a, b)\right\}$ 的点, 而这 一条件不是一般环上椭圆曲线均能满足的, 所以, 该 方案的使用在曲线选择方面受到较大限制. 而对于我 们所提出的 $C_{n}(a, b)$ 上的新方案, 定理 2 的推论保证 了环上圆雉曲线上一定存在阶为 $M_{n}$ 的基点, 推论 2 还给出了阶为 $M_{n}$ 的基点的一种获取方法. 因此, 在 曲线选择方面, $C_{n}(a, b)$ 比 $E_{n}(a, b)$ 上拥有更大的选 择范围.

因此, $C_{n}(a, b)$ 上的 RSA 型新方案可以获得比 $E_{n}(a, b)$ 上更好的密码特性.

\section{参考文献}

1 Koyama K, Maurer U, Okamoto T, et al. New public-key schemes based on elliptic curves over the ring $\mathbb{Z}_{n}$. In: Feigenbaum J eds. Advances in Cryptology-CRYPTO'91. Lect Notes Comput Sci. Santa Barbara: Springer-Verlag, 1992. 576: 252-266

$2 \mathrm{Qu} \mathrm{M} \mathrm{H}$, Vanstone S. On ID-based Cryptosystems Over $\mathbb{Z}_{n}$. 庆贺柯召院士九十寿辰暨国际数论学术研讨会上的报告. 成 都, 2000. 13-26

3 朱文余, 孙琦. 环 $\mathbb{Z}_{n}$ 上椭圆曲线及数字签名方案. 电子与信息学报, 2003, 25(增刊)：40-47

4 朱文余, 孙琦. 环 $\mathbb{Z}_{n}$ 上椭圆曲线的密钥交换协议. 电子学报, 2005, 33(1): 83-87

5 曹珍富. 基于有限域 $\mathbb{F}_{p}$ 上圆锥曲线的公钥密码系统. 刘木兰, 龚奇敏编. 密码学进展—CHINACRYPT 98. 北京: 科学 出版社, 1998.45-49

6 曹珍富. RSA 与改进的 RSA 的圆锥曲线模拟. 黑龙江大学自然科学学报, 1999, 16(4): 15-18

7 孙琦, 朱文余, 王标. 环 $\mathbb{Z}_{n}$ 上圆锥曲线和公钥密码协议. 四川大学学报 (自然科学版), 2005, 42(3): 471-478

8 王标, 朱文余, 孙琦. 基于剩余类环 $\mathbb{Z}_{n}$ 圆锥曲线的公钥密码体制. 四川大学学报 (工程科学版), 2005, 37(5): 110-116

9 孙琦, 张起帆, 彭国华. 计算群元的整数倍的一种算法及其在公钥密码体制中的应用. 王育民编. 密码学进展—CHINACRYPT 2002. 北京: 电子工业出版社, 2002. 117-124

10 Hastad J. On using RSA with low exponent in a public key network. In: Williams H C. eds. Advances in CryptologyCRYPTO 85. Lect Notes Comput Sci. Santa Barbara: Springer-Verlag, 1986. 218: 403-408

11 Wiener M J. Cryptanalysis of short RSA secret exponents. IEEE Trans Inf Theory, 1990, 36(3): 553-558

12 Boneh D. Twenty years of attacks on RSA cryptosystem. Notices of the AMS, 1999, 46(2): 203-213

13 孙琦, 彭国华, 朱文余等. 环 $\mathbb{Z}_{n}$ 上圆锥曲线 RSA 型公钥密码体系和抗小私钥 $\mathrm{d}$ 攻击. 王小云, 杨义先编. 密码学进展 -CHINACRYPT'2006，第九届中国密码学学术会议论文集. 北京: 中国科学技术出版社, 2006. 96-102

14 朱文余, 孙琦. 计算机密码应用基础. 北京: 中国科学技术出版社, 2007. 111-130 